\title{
Les conséquences géomorphologiques de l'occupation du sol et des changements climatiques dans un bassin-versant rural sahélien
}

Ibrahim Bouzou Moussa' ${ }^{1}$

Oumarou Faran Maiga ${ }^{2}$ Jean-Marie Karimou Ambouta ${ }^{3}$ Benoit Sarr ${ }^{5}$

Luc Descroix 6

Mahaman Moustapha Adamou ${ }^{4}$

${ }^{1}$ Département de géographie

Faculté des lettres et sciences humaines

Université Abdou-Moumouni

BP 418 Niamey

Niger

2 Département de géographie faculté des lettres et sciences humaines

Université Abdou Moumouni de Niamey BP 418 Niamey

Niger

<faranmaigaoumarou@yahoo.fr>

3 Département des sciences du sol

Faculté d'agronomie Université Abdou

Moumouni de Niamey

BP 10960 Niamey

Niger

<ambouta.karimou@yahoo.fr>

${ }^{4}$ Département de génie rural, eaux et forêts

Faculté d'agronomie Université Abdou

Moumouni de Niamey

BP 10960 Niamey

Niger

<adamou@refer.ne>

${ }^{5}$ Centre régional AGRHYMET

BP 11011 Niamey

Niger

<b.sarr@agrhymet.ne>

${ }^{6}$ Laboratoire d'étude des transferts

en hydrologie et environnement

BP 53

38041 Grenoble

France

<Luc.descroix@ird.fr>

Tirés à part : I. Bouzou Moussa

\section{Résumé}

Depuis 1994, le Kori Mountséka situé dans l'Adar Doutchi au centre-sud du Niger est redevenu fonctionnel sur $70 \mathrm{~km}$. Avant cette date, les écoulements étaient perturbés par des verrous constitués de dépôts de sable éolien et de cônes de déjection. Il se formait ainsi des mares, particulièrement dans la partie amont, alors que, dans la partie médiane et aval, le lit servait comme champ de culture de mil. Les observations directes sur le terrain ont permis de mettre en évidence que ce bassin-versant est soumis à une dynamique hydroérosive intense liée à l'érodibilité des sols et à la faiblesse du couvert végétal. Le traitement des données pluviométriques relève l'importance des extrêmes pluvieux. Enfin, les cartes de répartition spatiale des processus d'érosion hydrique en relation avec l'occupation des sols en 1986 et en 2000 confirment cet état de fait.

Mots clés : changement climatique, érosion, géomorphologie, Niger.

\section{Abstract}

\section{Geomorphological consequences of land use and climate change in a sahelian rural} catchment basin

Since 1994, the Mountseka valley in the Adar Doutchi area (south central Niger) has had $70 \mathrm{~km}$ of flowing water. Before this time, water flow was hindered by wind blown sand deposits and dejection cones. For this reason, particularly in its upstream section the valley had only ponds. The middle and downstream sections were used as fields for growing millet. Direct investigation has shown that this catchment basin is subject to an intensive process of water erosion due to the fragility of the soil and the weakness of ground vegetation. The processing of rainfall records shows the extent of rainfall extremes. Finally, maps showing the spatial layout of water erosion in relation to land occupation between 1986 and 2000 confirm our observations.

Key words: climate change, erosion, geomorphology, Niger.

D ans les milieux sahéliens et dans un contexte de très forte anthropisation, on assiste à une augmentation des écoulements sur les versants qui se traduit par:

- la modification des états de surface des sols ;
- le ravinement ;

- l'élargissement, voire l'approfondissement et l'ensablement des cours d'eau.

De nombreuses études [1-8] lient cette dynamique aux changements d'usages des sols. Cependant, elles ne font pas toujours cas des changements climatiques, et, 
notamment, d'une éventuelle amélioration de la pluviométrie dans I'augmentation des écoulements.

L'objectif de la présente étude est de comprendre la dynamique hydroérosive actuelle du kori ${ }^{1}$ Mountséka, de vérifier si l'amélioration de la pluviométrie observée depuis quelques années au Niger favorise les écoulements saisonniers dans un bassin-versant très anthropisé. En effet, le kori Mountséka situé dans le département de Birnin Konni au centre-sud du Niger se caractérise, depuis 1994, par un écoulement saisonnier sur $70 \mathrm{~km}$. Avant cette date, les écoulements alimentaient seulement des mares situées en amont. En d'autres termes, les questions sont les suivantes:

- quelles sont les causes d'un tel phénomène ? Une amélioration de la pluviométrie dans un contexte de forte anthropisation? Une augmentation des événements extrêmes?

- un processus de fin de l'endoréisme d'un bassin-versant sahélien? ou une simple coalescence des mares?

- quelles seront les conséquences hydrogéomorphologiques?

Après la présentation de la démarche et du milieu d'étude, cet article traite des résultats à travers la dynamique hydroérosive actuelle en relation avec l'occupation du sol, l'analyse de la pluviométrie de la station météorologique de Birnin Konni et, enfin, ouvre une brève discussion.

\section{Matériel et méthode}

En 2006 et 2007, nous avons effectué deux missions par année afin de caractériser le bassin-versant du kori. Pour ce faire, nous avons choisi plusieurs transects qui nous ont permis de faire cette caractérisation et de ressortir ainsi l'état actuel de la dynamique hydroérosive. Un GPS, un mètre ruban ainsi que des témoins naturels comme les arbres ont servi pour les mesures. Sur chaque transect et par unité morphopédologique ont été décrites la forme, la géologie, les formations superficielles ef leurs organisations pelliculaires superficielles, l'occupation du sol et la dynamique érosive. Des ravines ont été identifiées et suivies en mesurant le recul de têtes.

Deux cartes de répartition des processus d'érosion hydrique, en relation avec l'occupation du sol en 1986 et en 2000 , ont été réalisées à partir des images satellitales obtenues au Centre régional AGRHYMET de Niamey et traitées à I'aide des logiciels ERDAS et Arc View. Les images Landsat d'une résolution de

\footnotetext{
${ }^{1}$ Kori : cours d'eau à écoulements spasmodiques.
}

$30 \mathrm{~m}$ ont été déjà traitées au Centre régional AGRHYMET. La clé d'interprétation est celle proposée pour la nomenclature d'occupation du sol du Niger. La vérification a été faite le long des transects étudiés. Les surfaces sujettes à des processus d'érosion dominants ont été digitalisées, et à chacune d'elles ont été affectés des symboles correspondant au type d'occupation du sol.

La collecte des données pluviométriques journalières et annuelles de la station de Birnin Konni a été effectuée auprès de la direction de la météorologie nationale du Niger (DMN). La période d'étude 19612006 ne présente aucune lacune. Le logiciel Instat+ version 3. 030, logiciel d'analyse statistique de données agroclimatologiques et modèle de simulation agrométéorologique, mis au point par le Statistical Services Centre de l'université de Reading au Royaume-Uni, a servi pour déterminer les pluies journalières extrêmes annuelles. Ces données ainsi que les cumuls annuels ont été exportés sur Excel pour l'établissement des graphiques. L'analyse pluviométrique a été basée sur l'étude des cumuls annuels, de la moyenne mobile au pas de temps de cinq ans, de l'indice d'anomalie pluviométrique de Lamb, qui est la différence entre la pluviométrie annuelle et la pluviométrie moyenne divisée par l'écart-type de la série analysée (1961-2006) [16]. Cet indice nous a permis de mieux cerner, sur la série étudiée, les périodes sèches et humides. L'analyse des pluies maximales journalières a été effectuée à l'aide du logiciel SAFARHY 3.1-06/96 (Statistiques et analyse fréquentielle adaptées à l'évaluation du risque en hydrologie). Après la saisie des données et en fonction de leurs caractéristiques, le logiciel conseille les lois à retenir pour le traitement. Les pluies extrêmes étant en général distribuées selon la loi de Gumbel, nous avons utilisé la méthode du maximum de vraisemblance dans un intervalle de confiance à $95 \%$. Les temps de retour des pluies extrêmes de 1990 à 1994 et de l'année 2006 (années ayant enregistré les plus forts extrêmes) ont été déterminés. La lecture se fait de manière directe à partir d'une table de quantiles de probabilités et de temps de retour obtenu après le traitement des données. Pour une pluie maximale journalière correspond un quantile et, à défaut, on la situe entre deux quantiles en privilégiant le quantile inférieur en considérant la probabilité de non-dépassement.

\section{Milieu d'étude}

Le kori Mountséka est situé au centre-sud du Niger dans l'Adar (figure 1). Son bassin-versant est un sous-bassin-versant du Dallol Maouri, lui-même affluent du fleuve Niger. Sa plus grande partie se trouve en territoire nigérien dans les régions de Tahoua (département de Birnin Konni) et de Dosso (département de Doutchi). Une autre partie concerne le Nord Nigéria où le kori fait une boucle avant de retraverser le territoire nigérien pour se jeter dans le Dallol Maouri.

D'une superficie de $5505 \mathrm{~km}^{2}$, le bassinversant présente une forme allongée et des pentes relativement faibles.

Dans ce bassin-versant affleurent les grès ferrugineux peu résistants du continental terminal.

Les formes dégagées témoignent d'une paléomorphogenèse intense. Cette dynamique s'est traduite par la formation de dunes. On distingue quatre unités morphodynamiques (figure 2) :

- les sommets de plateaux et des buttes cuirassées situés à une altitude moyenne de $370 \mathrm{~m}$ au nord-est et $300 \mathrm{~m}$ à l'ouest, et leurs talus d'éboulis courts concaves à convexoconcaves profondément ravinés laissant affleurer les grès argileux altérés ; - deux glacis rocheux anciens très courts, à surface cuirassée, taillés dans les grès argileux; un glacis plus récent limonosableux à sablolimoneux qui peut être étendu ou court ;

- les dunes orientées Est-Ouest qui fossilisent de grandes surfaces des sommets et de la vallée traduisent une phase sèche et expliquent l'endoréisme du réseau hydrographique. La fossilisation des lits des koris s'est faite progressivement par le mouvement des sables des versants;

- le kori orienté NNE-SSO, à tracé sinueux avec des ombilics occupés par des mares et des verrous constitués par des cônes de déjection des ravines latérales, présente un lit à fond plat sableux, argilosableux ou limonoargileux.

Le climat est de type sahélien. Ainsi, sur la période 1961-2006, Birnin Konni, la plus ancienne et la plus proche station, a une pluviométrie moyenne annuelle de $494,9 \mathrm{~mm}$.

L'agriculture et l'élevage, tous les deux de types extensifs, constituent les principales activités des populations. S'y développe aussi le commerce de bois à travers les marchés ruraux dans quelques villages. Du fait de la forte anthropisation de l'ensemble du bassin-versant, sur toutes les unités, la végétation climatique a disparu. II ne subsiste que des ligneux bas de combretacées, avec un recouvrement très faible dans les champs, moyen dans les jachères et moyen à fort le long de certaines ravines et dans les dépressions fermées. 


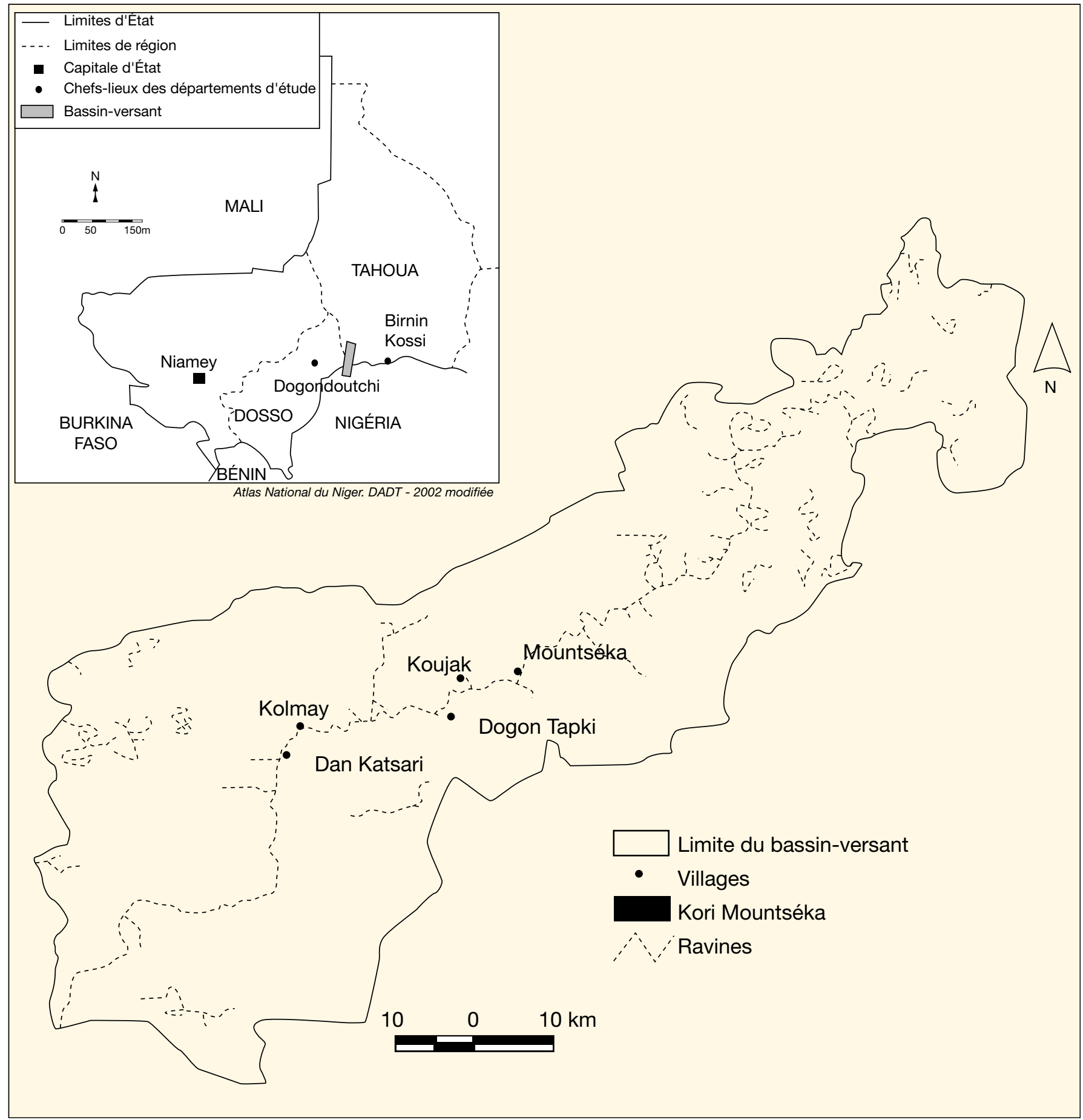

Figure 1. Localisation de la zone d'étude.

\section{Résultats}

Dynamique hydroérosive actuelle en relation avec celle de l'occupation du sol

La dynamique hydroérosive actuelle se caractérise par des retouches des formes héritées dont les plus actives sont le décapage et le ravinement. La carte de réparti- tion des processus d'érosion hydrique en relation avec l'occupation du sol en 1986 et en 2000 (tableau 1 et figure 3) illustre bien l'augmentation des surfaces affectées par les différents types de ruissellement.

Ainsi, sur les sommets de plateaux et des buttes à brousse tigrée dégradée, le ruissellement est diffus et lent, $d^{\prime}$ 'où une faible érosion hydrique. En effet, sur ces sommets affectés d'une pente voisine de $1 \%$, le ruissellement diffus décape peu. La prédominance des croûtes de décantation témoigne de ce type de ruissellement.

Sur les glacis indurés à brousse mouchetée à pente plus forte de 5 à $10 \%$, la mise en culture favorise la formation des surfaces de déflation à croûtes d'érosion (figure 4) qui occupent 50 à $60 \%$ de ces unités. $\|$ s'y développe ainsi un fort ruissellement 


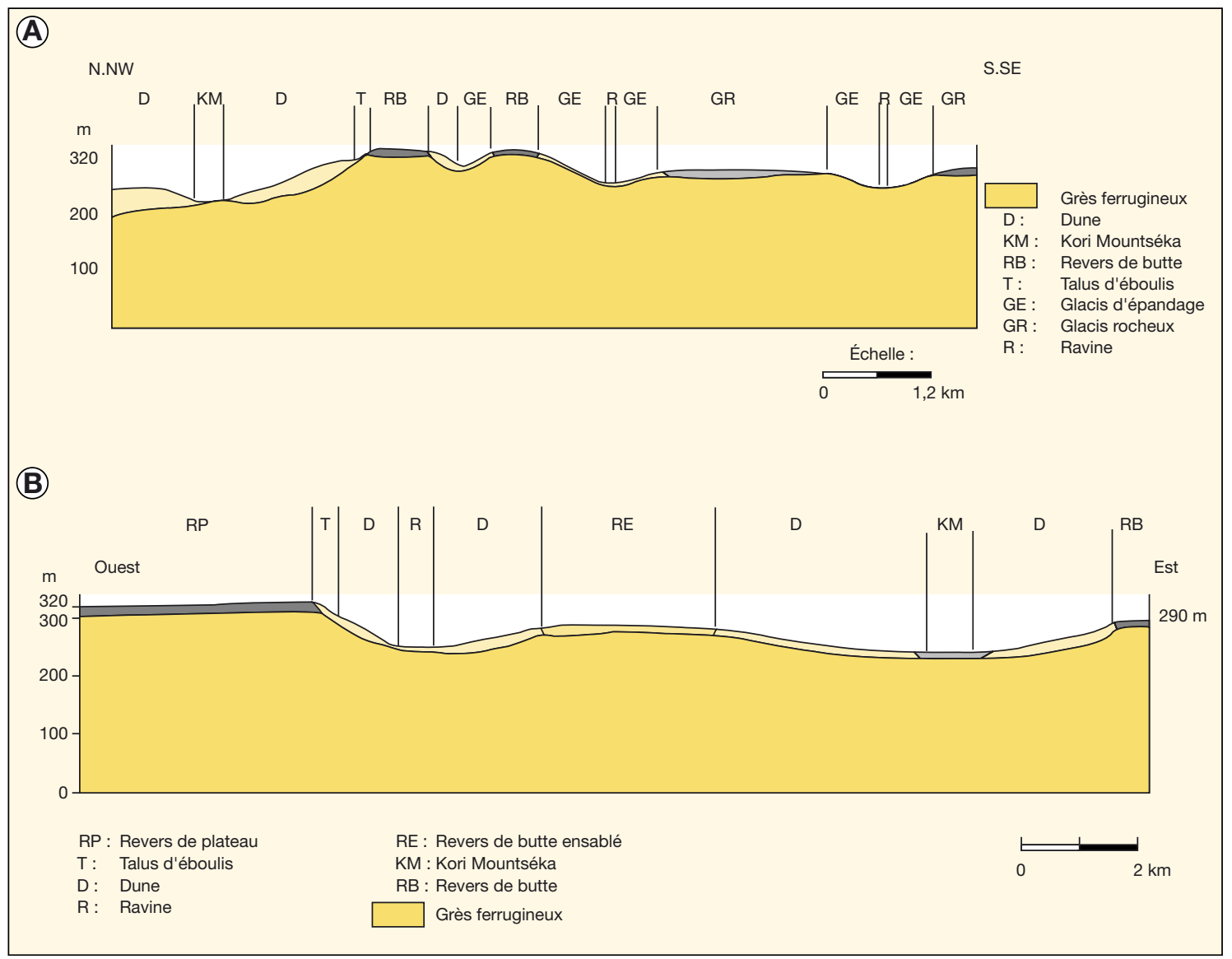

Figure 2. Profils transversaux de la vallée du kori Mountséka.

A) secteur médian ; B) secteur aval. Kori : cours d'eau à écoulements spasmodiques.

diffus avec un décapage intense des microhorizons superficiels donnant lieu à des micromarches d'escaliers caractéristiques. La brousse mouchetée passe de 138135,5 à 94251,8 hectares en 15 ans, soit un taux de réduction annuel de $2,11 \%$; quant aux zones de cultures pluviales, leur superficie évolue de 221068,8 à 262601,3 hectares, soit aussi un taux d'accroissement annuel de $1,25 \%$.

Les surfaces affectées par le ruissellement concentré, phénomène le plus remarquable, concernent les terrains à pentes sensibles à broussailles ou champs abandonnés: les talus des plateaux et des buttes, les glacis indurés et les bas-fonds des koris affluents. On observe là également un changement notable: ces superficies ont varié de 111341,10 à 113575,55 ha, soit un taux d'accroissement de $2 \%$.

Cette période a été aussi marquée par le développement de ravines latérales (figure 3) qui forment des cônes de déjection à la confluence des koris. Sur ces terrains et particulièrement sur les glacis sableux, I'action du ruissellement concentré est spectaculaire. Les écoulements linéaires provoqués par de grosses averses creusent de nombreuses ravines qui, par érosion régressive, prolongent leur réseau vers l'amont en développant des ramifications de têtes de recul. Le recul de tête peut être important, à l'exemple de la ravine de Mountséka créée par une pluie en 2006, et dont les têtes droite et gauche ont reculé respectivement, en 2007, de 22,4 et $11,5 \mathrm{~m}$ (figure 5). Par ailleurs, de nombreux bas-fonds affluents du kori Mountséka, naguère stables, sont aujourd'hui affectés par la reprise d'une intense érosion verticale. Les petits koris creusent leur fond à des mètres de profondeur et sapent de plusieurs mètres les berges sableuses généralement mises en culture. De la sorte, selon les témoignages oraux, beaucoup de paysans ont perdu leurs champs de bas-fonds. Les écoulements torrentiels charrient d'énormes matériaux solides qui sont déposés soit dans certaines mares localisées dans le lit du kori Mountséka (Aléla, Baizo, Koujak), soit répandus à la confluence sous forme de cônes de déjection.

Tableau I. Évolution des superficies affectées (en ha) par les types de processus d'érosion hydrique dans le bassin-versant du kori Mountséka en 1986 et en 2000.

\begin{tabular}{|lccccc|}
\hline & Ac & Rdl & Rdm & Rdf & Rc \\
\hline 1986 & 5173,2 & 29694,0 & 138135,5 & 221066,6 & 111341,1 \\
2000 & 4321,8 & inchangé & 94251,8 & 262427,8 & 114094,3 \\
\hline
\end{tabular}

Ac : accumulation; Rdl : ruissellement diffus lent; Rdm : ruissellement diffus moyen ; Rdf : ruissellement diffus fort ; Rc : ruissellement concentré. Kori : cours d'eau à écoulements spasmodiques. 


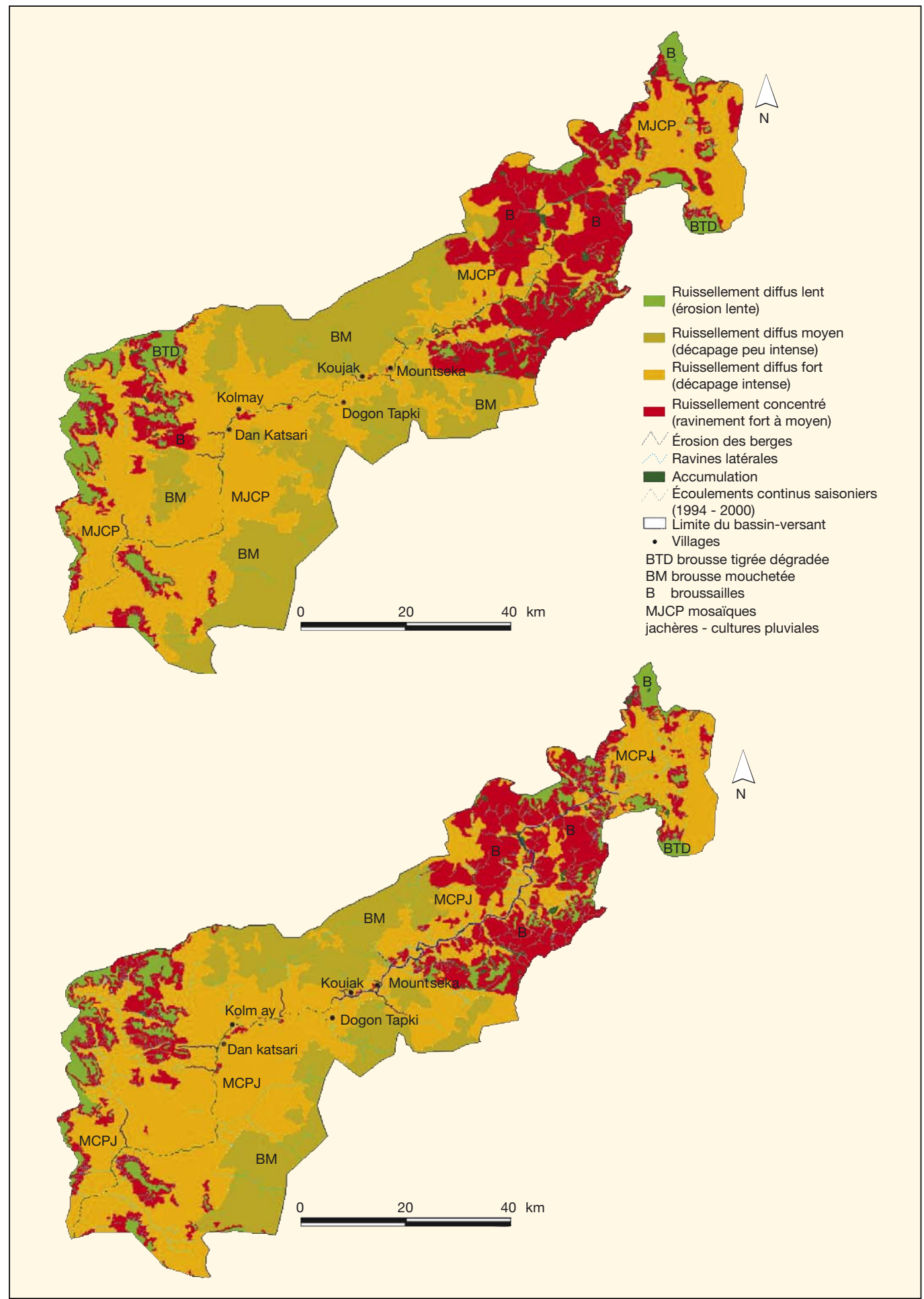

Figure 3. Répartition spatiale des processus d'érosion hydrique en relation avec l'occupation du sol dans le bassin-versant du kori Mountséka en 1986 et en 2000.

Kori : cours d'eau à écoulements spasmodiques.

Cette dynamique explique la reprise progressive de la fonctionnalité de ce kori : d'abord depuis 1994 de l'amont au niveau de Kwara jusqu'à Koujak, puis Dogon Tapki en 2005 et 2006 et Kolmay en 2007. Vers la fin du mois d'août, il se forme désormais un long plan d'eau continu large et profond. À la fin de l'hivernage, il s'interrompt par endroits par quelques seuils. L'eau y persiste dans de grandes mares jusqu'à la prochaine saison des pluies.
Analyse de la pluviométrie de la station de Birnin Konni

Les figures 6 et 7 montrent l'évolution de la pluviométrie à Birnin Konni de 1961 à 2006. II ressort une variabilité interan- 


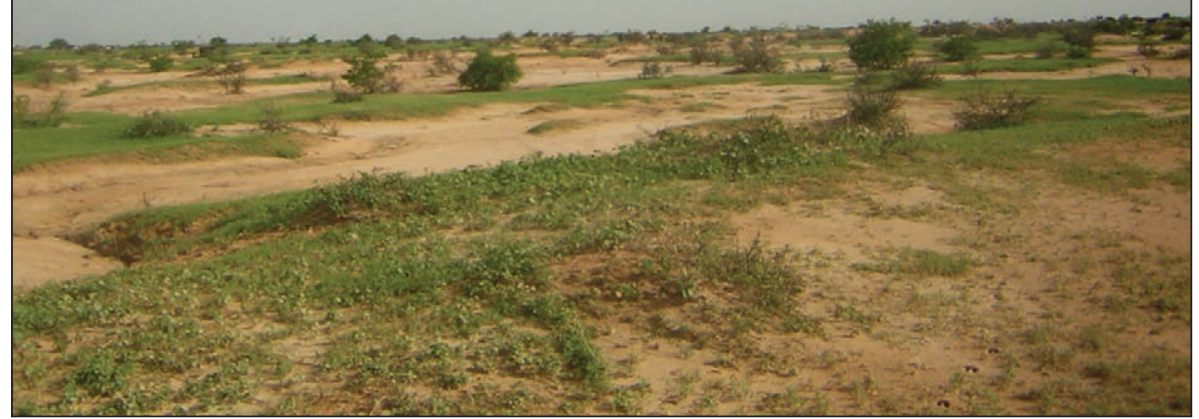

Figure 4. Développement de surfaces de déflation et de ruissellement sur un glacis induré dans le secteur médian de la vallée du kori Mountséka.

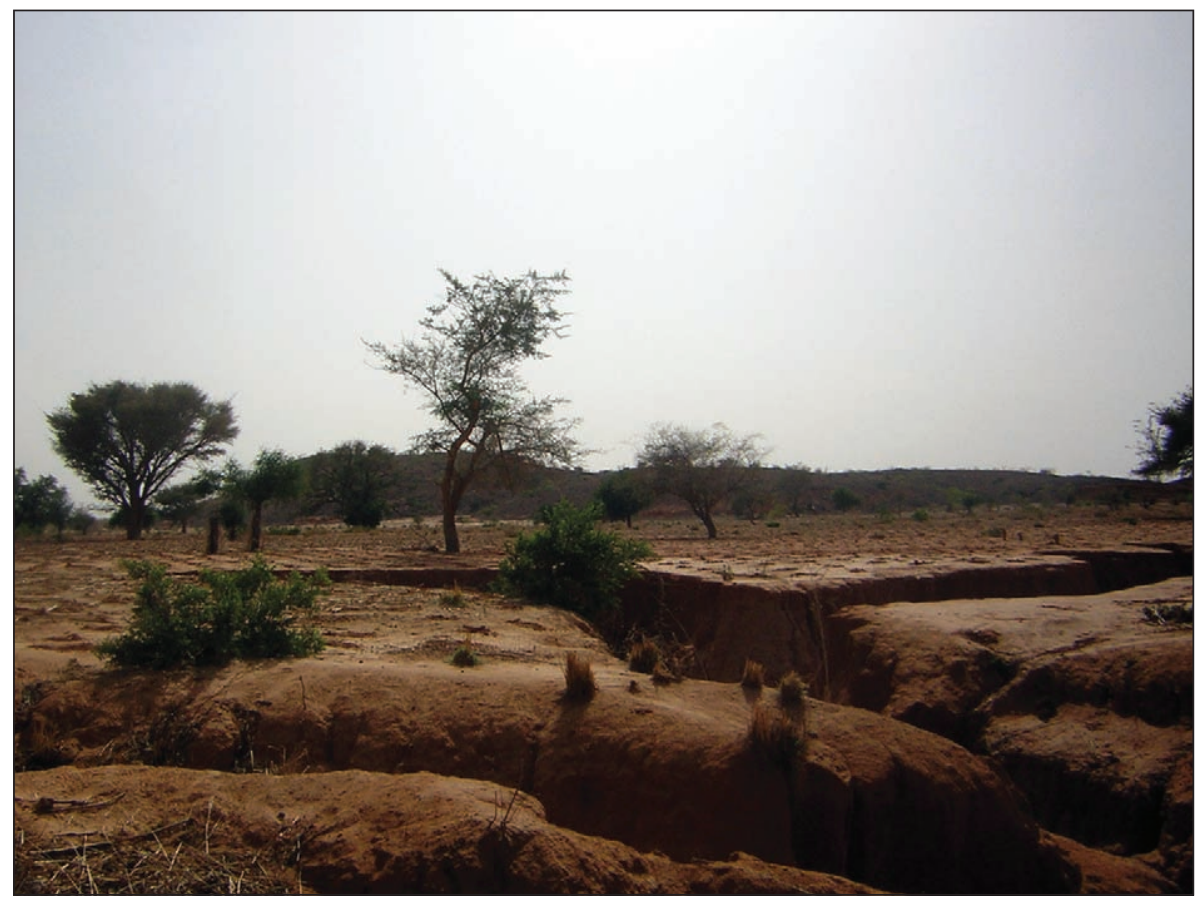

Figure 5. Ravine latérale créée par une pluie en août 2006.

nuelle des précipitations, caractéristiques du climat sahélien. Ainsi, en considérant la moyenne sur cette période, 22 années sont déficitaires contre 24 années moyennes ou excédentaires. Les moyennes mobiles sur cing ans et l'indice d'anomalie de Lamb [16] permettent de distinguer quatre périodes humides: 1961-1969, 1976- quatre ans, de 2000 à 2003. Si la période 1961-1969 a été plus régulière avec sept années consécutives humides, c'est la période 1989-1999 qui a connu le plus d'années excédentaires et des extrêmes journaliers plus importants. De 1986 à 1994, ont été enregistrés des extrêmes journaliers compris entre 51 et $109 \mathrm{~mm}$. On observe également le même phénomène en 2006, année excédentaire avec un cumul de $775,9 \mathrm{~mm}$ et un extrême journalier de 121,7 mm. Le tableau 2 illustre le temps de retour de ces extrêmes journaliers. Ainsi, la pluie de 109 mm, tombée le 5 août 1994, a un temps de retour de 125 ans. II est tombé cette année $733,1 \mathrm{~mm}$, et les écoulements dans le kori principal ont atteint le village de Mountséka. Quant à la pluie de $121,7 \mathrm{~mm}$ tombée le 10 septembre 2006 , son temps de retour est de 200 ans. L'amélioration de la pluviométrie ces dernières années ainsi que l'augmentation des extrêmes journaliers contribuent à l'accroissement des écoulements et de l'érosion ainsi qu'à la reprise de la fonctionnalité progressive du Kori. En effet, les surfaces de déflation à croûtes d'érosion qui se développent sur les glacis ef les versants de dunes favorisent le ruissellement et l'érosion.

\section{Discussion}

Les images satellitales de 1986 et de 2000 ainsi que les investigations sur le terrain révèlent que le bassin de Mountséka a subi une profonde mutation de l'occupation du sol. Celle-ci s'est traduite par une extension des aires de cultures aux dépens du couvert végétal susceptible de protéger le sol contre l'érosion. Dans ce contexte de dénudation des unités hydromorphopédologiques, on assiste à une augmentation des écoulements tant sur les versants que dans les koris. De nombreuses études confirment le rôle des changements d'usage des sols sur le ruissellement et l'érosion [2-12]. Ailleurs, Tribak et Morel ont montré le rôle de l'occupation des sols sur le développement du ravinement [13].

Mais l'intérêt du développement de ce phénomène réside non seulement dans le rôle de la forte anthropisation observée dans les milieux soudanosahéliens depuis les années 1950 [14], et des extrêmes pluvieux que l'on enregistre en saison déficitaire comme en saison excédentaire [9], mais aussi dans le fait que ces extrêmes, ces dernières années, sont nettement plus marqués (figure 4). Ầ cela, il faut ajouter le rôle très important que jovent les pluies maximales journalières en $n$ jours consécutifs dans 


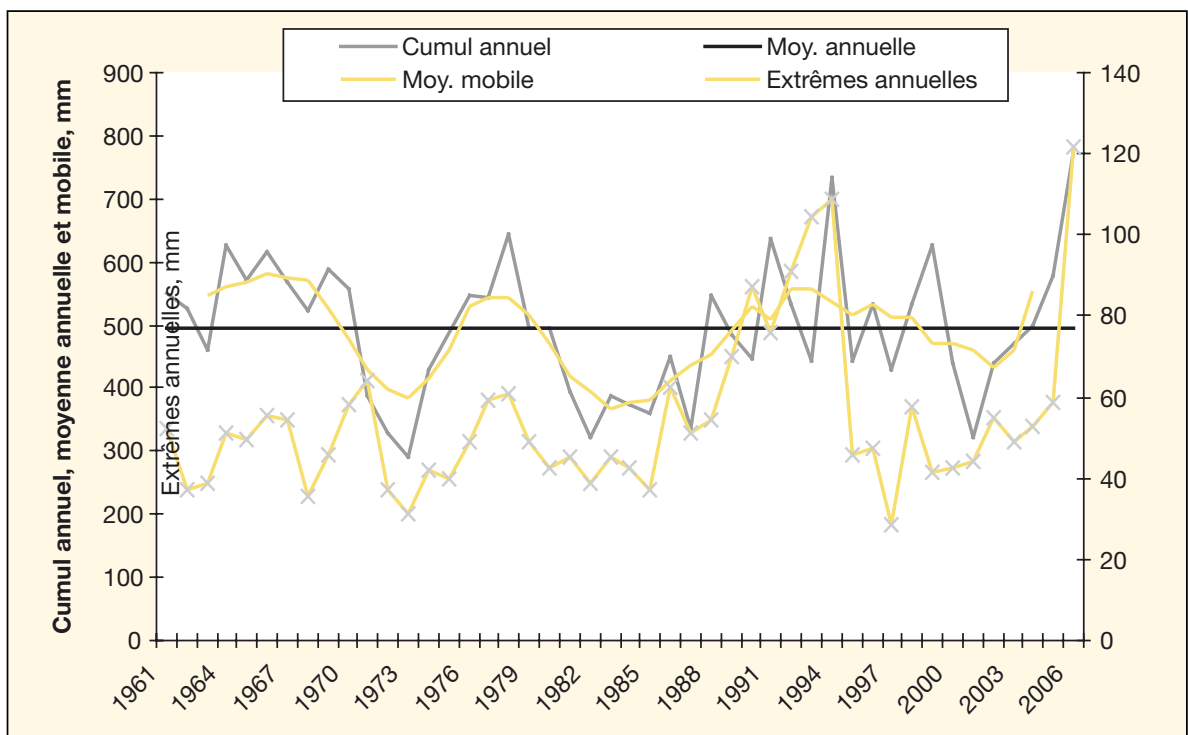

Figure 6. Évolution de la pluviométrie à Birnin Konni (1961-2006).

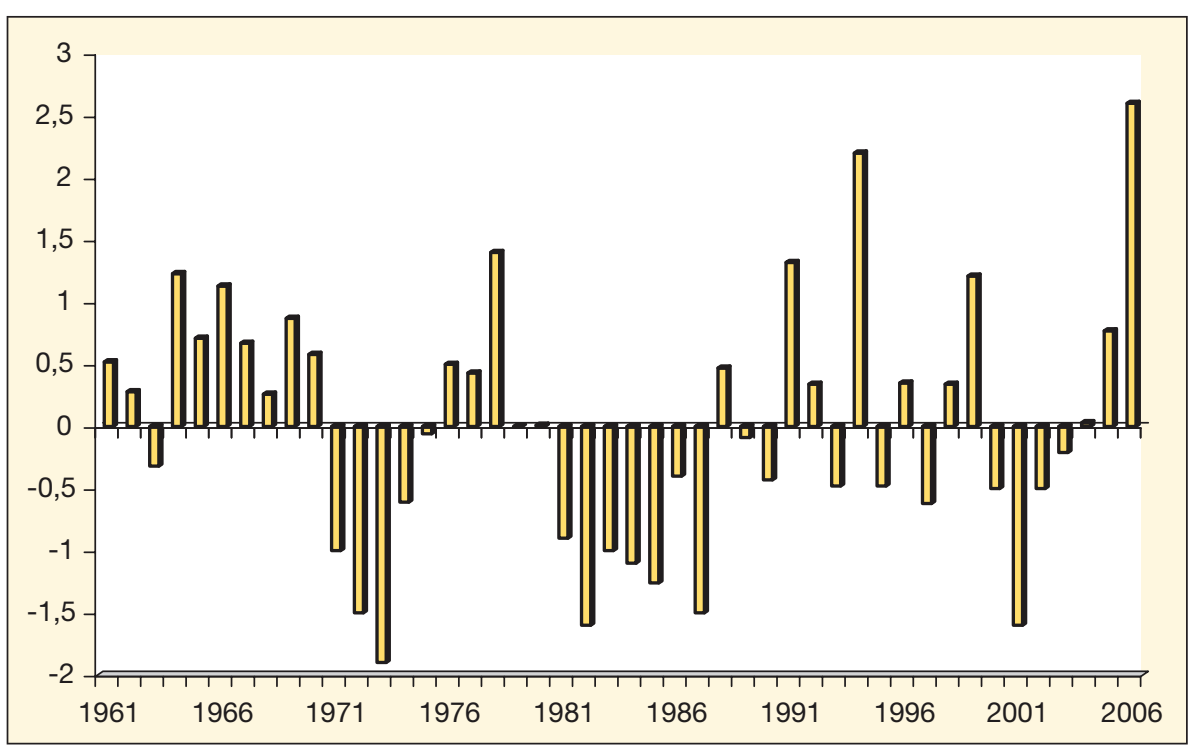

Figure 7. Évolution des précipitations à Birnin Konni de 1961 à 2006 exprimée en fonction de l'indice d'anomalie pluviométrique de Lamb (1982).

l'augmentation des ruissellements et de l'érosion [15]. Aussi, au vu de ces caractéristiques pluviométriques, ne peut-on pas cette reprise de la fonctionnalité du kori Mountséka traduitelle le début du processus de fin de l'endoréisme des bas-fonds sahéliens, ou est-ce une simple coalescence de mares?

En effet, dans le même contexte de retour de pluviométries plus abondantes et de forte anthropisation, on observe le même phénomène dans d'autres régions du Niger, le cas du kori Mountséka n'étant pas isolé. Dans la région de Maradi située au centre-sud du Niger, depuis quelques années, le Goulbin Kaba coule en saison humide. Le Kori Ouallam, affluent du fleuve Niger qui prend sa source au Mali, a repris progressivement ses écoulements saisonniers depuis 1999 pour dépasser, en 2006, la ville de Ouallam située à 100 km au Nord de Niamey. La même année, un des affluents du Kori Ouallam, le kori Goubé situé à la périphérie de Niamey a aussi coulé tout le long de son cours. Les conséquences d'un tel phénomène sont $d$ 'ordres hydromorphomopédologiques et socioéconomiques. Les écoulements deviennent plus importants sur des formations superficielles dénudées, avec comme corollaire une érosion de forte intensité des versants et l'ensablement du fleuve par les koris affluents, mais aussi, dans ces milieux endoréiques, la recharge de la nappe [17].

S'il est vrai que l'érosion décape les formations superficielles, un retour progressif à une pluviométrie normale suppose une bonne production céréalière sur les terres non dégradées et l'utilisation agrosylvopastorale des nombreux plans d'eau qui se forment. Les perceptions des populations de cette nouvelle dynamique divergent selon les villages. En amont, dans des villages comme Aléla, Baizo, la disparition des mares a entraîné une perte en terres de cultures de décrue. L'arboriculture s'est, en revanche, maintenue sur les surfaces inondables. En aval, les terres de basfonds ensablées qui, jusque-là, étaient utilisées comme champs de cultures pluviales de mil sont occupées aujourd'hui par les eaux. Il faut attendre leur retrait pour entreprendre des cultures maraîchères qui se sont développées ces dernières années,

Tableau II. Période de retour (en années) de quelques pluies maximales journalières à la station de Birnin Konni (Niger).

\begin{tabular}{|c|c|c|c|c|c|c|}
\hline \multirow[t]{2}{*}{ Date } & \multirow[t]{2}{*}{ Pluies maximales journalières (mm) } & \multirow[t]{2}{*}{ Quantiles (mm) } & \multirow[t]{2}{*}{ Probabilités } & \multirow[t]{2}{*}{ Période de retour } & \multicolumn{2}{|c|}{ Intervalle de confiance à $95 \%$} \\
\hline & & & & & Borne inférieure & Borne supérieure \\
\hline 15 mai 1990 & 87,2 & 83,88 & 0,9500 & 20 & 73,62 & 94,14 \\
\hline 17 septembre 1991 & 75,8 & 74,84 & 0,9000 & 10 & 66,45 & 83,23 \\
\hline 21 août 1992 & 91,1 & 83,88 & 0,9500 & 20 & 73,62 & 94,14 \\
\hline 9 août 1993 & 104,4 & 104,35 & 0,9900 & 100 & 89,68 & 119,02 \\
\hline 5 août 1994 & 109,0 & 107,16 & 0,9920 & 125 & 91,88 & 122,45 \\
\hline 10 septembre 2006 & 121,7 & 113,08 & 0,9950 & 200 & 96,50 & 129,67 \\
\hline
\end{tabular}


en plus de l'arboriculture. Enfin, l'aspect positif, unanimement reconnu par les populations des villages situés en aval, est I'accessibilité à l'eau. À titre d'illustration, les populations de Kan Guiwa disposent aujourd'hui de l'eau en permanence, alors qu'elles devaient aller la chercher à Dogon Tapki ou à Mountséka distants de $6 \mathrm{~km}$. Tout le long de ce tronçon, la conduite des animaux aux puits ou à des plans d'eau n'est plus un calvaire.

Néanmoins, ces phénomènes s'observent dans des bassins-versants non aménagés. Que se passe-t-il dans les bassins-versants des koris comme ceux de l'Adar fortement aménagés qui se jettent tous dans le Dallol Maouri affluent du fleuve Niger? Si une telle dynamique se maintenait, ne va-t-on pas assister à des écoulements avec des charges solides jusqu'au fleuve déjà menacé d'ensablement? Toute situation qui interpelle les acteurs du développement rural pour le suivi de tel phénomène et la recherche de solution à la dynamique hydroérosive qui se crée. Cependant, dans ces milieux semi-arides où les processus hydriques et éoliens interagissent, il est aussi possible que l'eau et le vent qui entaillent des formations sableuses dénudées recréent des verrous par accumulation de sable qui favorisent la formation des mares. Verrous qui peuvent être disséqués à l'occasion d'événements pluvieux exceptionnels.

\section{Conclusion}

La présente étude met en évidence le rôle de l'occupation du sol et de l'amélioration de la pluviométrie, et des extrêmes pluvieux sur la dynamique hydroérosive intense observée ces dernières années sur un bassin-versant endoréique. Entre 1986 et 2000, l'augmentation des superficies cultivées s'est accompagnée de celle des processus de ruissellement diffus fort et concentré.

Cet accroissement des processus de ruissellement explique l'importance des écoulements saisonniers sur $70 \mathrm{~km}$ dans le kori de Mountséka et la reprise de l'érosion verticale particulièrement dans les petits koris affluents. Il est certain que le processus de dénudation des sols va se poursuivre aussi longtemps que perdurera le système traditionnel de cultures extensives sans protection des sols. L'analyse de la pluviométrie montre une tendance à l'amélioration observée à partir des années 1990. Cela se traduit par des extrêmes pluvieux, comme ce fut le cas en 1994 où le phénomène a commencé à attirer l'attention. Cependant, rien n'autorise à affirmer que la reprise progressive de l'écoulement saisonnier dans le kori marque la fin de l'endoréisme. En effet, l'amélioration de la pluviométrie durant seulement deux décennies ne suffit pas pour mettre en évidence une tendance continue de la pluviosité. Toutefois, de par ses conséquences, une telle dynamique, si elle se maintenait, mérite d'être suivie.

\section{Références}

1. Desconnets JC. Typologie et caractérisation hydrologique des systèmes endoréiques en milieu sahélien (degré carré de Niamey, Niger). Thèse de doctorat, université de Montpellier, 1994.

2. Shinen T. Recent accelerated gully erosion and its effects in dry savanna, southwest of Niger. In : Hori N, ed. Human response to drastic change of environments in Africa I. Tokyo: Metropolitan University, 1999.

3. Saadou Y, Mahamadou M. Impacts des pluies exceptionnelles sur l'environnement et les infrastructures socio-économiques; cartographie des zones à risques et propositions d'aménagement : cas du secteur Lélihibéri-Ganguel sur la rive droite aux abords de Niamey. Mémoire de maîtrise, département de géographie de la faculté des lettres et sciences humaines, université Abdou Moumouni de Niamey (Niger), 2000.

4. Mamadou I. Dégradation des bas-fonds ef stratégies adaptatives paysannes: cas du las Madarounfa et son bassin d'alimentation d'alimentation (Région de Maradi). Mémoire de maîtrise, département de géographie de la faculté des lettres et sciences humaines, université Abdou Moumouni de Niamey (Niger), 2001.

5. Mamadou I. Érosion et ensablement dans les koris du Fakara-Degré carré de Niamey, Niger. Mémoire de DEA, département de géographie de la faculté des lettres et sciences humaines, université Abdou Moumouni de Niamey (Niger), 2005.

6. Moussa MS. Genèse et morphodynamique actuelle des bas-fonds sahéliens : caractérisation du bas-fond Goubé dans le degré carré de Niamey. Mémoire de maîtrise, département de géo- graphie de la faculté des lettres et sciences humaines, université Abdou Moumouni de Niamey (Niger), 2004.

7. Abba B. Genèse et morphodynamique actuelle des bas-fonds sahéliens : caractérisation des basfonds de Birnin Lokoyo, Doutchi et Sormo (Région de Dosso, Niger). Mémoire de maîtrise, département de géographie de la faculté des lettres et sciences humaines, université Abdou Moumouni de Niamey (Niger), 2006.

8. Malam Abdou M. Genèse et morphodynamique actuelle des bas-fonds sahéliens : étude comparative de quelques bas-fonds ruraux de socle et de bassin sédimentaire de l'ouest nigérien. Mémoire de maîtrise, département de géographie de la faculté des lettres et sciences humaines, université Abdou Moumouni de Niamey (Niger), 2006

9. Faran MO. La dynamique actuelle dans le Zarmaganda. Recherches géomorphologiques dans l'Ouest nigérien. Thèse de Doctorat de $3^{\mathrm{e}}$ cycle de géographie physique, université Cheikh Anta Diop (Sénégal), 2000.

10. Bouzou MI. L'érosion dans la vallée de Keita. Contribution géomorphologique. Thèse unique, université Joseph Fourier Grenoble I, 1988.

11. Bouzou MI. Réponses géomorphologiques à la problématique de la conservation des eaux et des sols au Niger. Rapport d'habilitation à diriger des recherches, université Joseph Fourier Grenoble I, 2000

12. Bouzou MI. Impacts irréversibles sur l'environnement des pluies exceptionnelles au Niger. Ann Univ Abdou Moumouni 2006 ; VIII-B : 81-93.

13. Tribak A, Morel A. L'utilisation des terres et le ravinement dans les moyennes montagnes du prérif oriental (Maroc). Bull Res Erosion 2004 ; 23 : 236-47.

14. Roose É. Problèmes posés par l'aménagement des terroirs en zone soudano-sahélienne d'Afrique Occidentale. In: Aménagements hydro-agricoles et systèmes de production. Documents Systèmes Agraires, no 6. Montpellier: Cirad, 1987.

15. Mietton M. Dynamique de l'interface lithosphère-atmosphère au Burkina. Contribution géomorphologique à l'échelle de l'érosion en zone tropicale de savane. Thèse de doctorat d'État, université Grenoble I, 1988.

16. Ozer P, Bodart C, Tychon B. Analyse climatique de la région de Gouré, Niger oriental : récentes modifications et impacts environnementaux. CyberGeo: European Journal of Geography $2005 ; 308: 1-24$

17. Favreau G. Caractérisation et modélisation d'une nappe phréatique en hausse au Sahel. Thèse, université Paris XI, 2000. 